\title{
Assessment of the ecological and trophic status of Lake Bafa (Turkey) based on phytoplankton
}

\section{Bafa Gölü'nün (Türkiye) ekolojik ve trofik durumunun fitoplankton bazlı değerlendirilmesi}

\author{
Atakan Sukatar ${ }^{1}$ - Alperen Ertaş2* $\bullet$ Rıza Akgül ${ }^{3}$ \\ 1 Department of Biology, Faculty of Science, Ege University, 35100 Bornova, İzmir, Turkey \\ 2 Department of Biology, Faculty of Science, Ege University, 35100 Bornova, Izmir, Turkey \\ ${ }^{3}$ Department of Crop and Animal Production, Mehmet Akif Ersoy University, Burdur, Turkey \\ ${ }^{4}$ Department of Biology, Faculty of Science, Ege University, 35100 Bornova, İzmir, Turkey
} Inci Tüney Kızılkaya ${ }^{4}$

Sukatar, A., Ertaş, A., Akgül, R. \& Tüney KIzlkaya, I. (2021). Assessment of the ecological and trophic status of Lake Bafa (Turkey) based on phytoplankton Ege Journal of Fisheries and Aquatic Sciences, 38(2), 135-146. DOI:10.12714/egejfas.38.2.01

Abstract: Phytoplankton groups are one of the major quality element to be used in the evaluation of the trophic and ecological state of freshwater ecosystems according to the EU Water Framework Directive. This research was made to assess the trophic and ecological status of Lake Bafa in Turkey, on the basis of phytoplankton communities. Büyük Menderes River is one of the most important factor that carries pollutants to Lake Bafa. The eight sampling station were assigned to evaluate the ecological and trophic state of the lake. Phytoplankton species were collected monthly for 2 years study period. Most commonly used phtoplankton indices Q index and Carlson's Trophic State Index (TSI), and different versions of diversity indices were used to estimate trophic and ecological state of the lake. Similarities between the sampling stations were clustured by using the unweighted pair group method using arithmetic average (UPGMA), based on phytoplankton communities. Correlations between the applied indices were determined by using Pearson Correlation. After the identification of collected phytoplanktons, total of 63 taxa which belong to classis of Cyanophyceae $(11.2 \%)$, Bacillariophyceae $(49.2 \%)$, Chlorophyceae $(23.8 \%)$, Xanthophyceae (1.5\%), Euglenophyceae $(11.2 \%)$ and Dinophyceae (3.1\%) were detected. The $1^{\text {st }}$ and $2^{\text {nd }}$ stations were the most similar stations to each other $(88 \%)$ according to phytoplankton communities. Secchi disc depth (SD) and TP played an important role in the distribution of phytoplankton species in Lake Bafa. The highest significant positive correlation was determined between $Q$ and TSI $(r=0.987, p<0.01)$. Considering the TDI values in the phytoplankton composition of the lake, it can be said that although the productivity status of the studied lake is still "mesotrophic", it has a tendency towards "eutrophic" state. According to the $Q$ values, the first five stations reflect the moderate ecological state, while the $6^{\text {th }}, 7^{\text {th }}$ and $8^{\text {th }}$ stations represent the poor ecological state.

Keywords: Lake Bafa, phytoplankton, Q index, Trophic State Index, Water Framework Directive

Öz: Fitoplankton, AB Su Çerçeve Direktifi'ne göre tatlı su ekosistemlerinin trofik ve ekolojik durumunun değerlendirimesinde kullanılan en önemli kalite unsurlarından biridir. Bu araşıırma, Bafa Gölü'nün trofik ve ekolojik durumunu fitoplankton grupları temel alarak değerlendirmek için yapılmıştır. Büyük Menderes Nehri, kirleticileri Bafa Gölü'ne taşıyan en önemli faktörlerden biridir. Gölün ekolojik ve trofik durumunu değerlendirmek için sekiz örnekleme istasyonu belirlenmiş̧tir. Fitoplankton türleri 2 ylllık çalışma süresi boyunca aylık olarak toplanmış̧ır. Gölün trofik ve ekolojik durumunu tahmin etmek için en yaygın kullanılan fitoplankton indeksleri Q indeksi ve Carlson'un Trofik Durum Indeksi (TDI) ve çeşitllik indekslerinin farklı versiyonları kullanılmıştr. Uygulanan indeksler arasındaki korelasyonlar Pearson Korelasyonu kullanılarak belirlenmiştir. Örnekleme istasyonları arasındaki benzerlikler, fitoplankton topluluklarına dayanan aritmetik ortalama ile ağırlıksız çift grup metodu (UPGMA) kümeleme yöntemiyle belirlenmiştir. Toplanan fitoplankton örneklerinin tanımlanmasından sonra, Cyanophyceae (11.2\%), Bacillariophyceae (49.2\%), Chlorophyceae (23.8\%), Xanthophyceae (1.5\%), Euglenophyceae (11.2\%) ve Dinophyceae $(3.1 \%)$ sınıflarına ait toplam 63 takson tespit edilmiş̧tir. Fitoplankton topluluklarına göre 1. ve 2. istasyonlar (\%88) birbirlerine en yakın istasyonlar olduğu görülmüştür. Secchi disk derinliği (SD) ve TP Bafa Gölünde fitoplankton türlerine dağııımında önemli rol oynamıştı. En yüksek anlamlı pozitif korelasyon Q ve TDI arasında belirlenmiştir $(r=0.987, p<0.01)$. Gölün fitoplankton dağılımındaki TDI değerleri göz önüne alındığında, incelenen gölün verimlilik durumu halen "mezotrofik" olmakla birlikte, "ötrofik" duruma doğru bir eğilim gösterdiği söylenebilir. $Q$ değerlerine göre, ilk beş istasyon orta ekolojik durumu yansitırken, 6 ., 7. ve 8 . istasyonlar kötü ekolojik durumu temsil etmektedir.

Anahtar kelimeler: Bafa Gölü, fitoplankton, Q indeksi, Trofik Durum İndeksi, Su Çerçeve Direktifi

\section{INTRODUCTION}

Increasing human population cause big pressure on the aquatic ecosystems. The oceans, lakes, rivers and streams are being "squeezed" by human activities such as demotechnical and industrial improvements, agricultural activities and other human impact. Thus, ecological quality associated with water quality deteriorates (Vollenweider and Kerekes, 1982).
The European Union (EU) Water Framework Directive (WFD) 2000/60/EC commits EU member states and is adopted in 2000 takes a pioneering approach to protecting aquatic systems. The directive aime to achieve good qualitative and quantitative status of all surface water bodies and groundwaters (European Commission, 2000). 
According to the WFD, phytoplankton groups occupies an important place and it has a vital role in the aquatic systems, therefore, phytoplankton is one of the biological quality indicators required for evaluation of ecological status of freshwaters in Europe. Amount of the nutrient load, has direct effect on the community of the phytoplankton. Therefore many researchers have preferred, the usage of phytoplankton for both water quality and eutrophication impact assessment (Pasztaleniec and Poniewozik, 2010). In some cases, the accumulation of extra phytoplankton can cause undesirable situations such as decreasing the photosynthesis of submerged vegetation due to shading, and develop of anoxic conditions in aquatic ecosystems (Pasztaleniec, 2016). As a result of this kind of situations, phytoplankton composition shows alterations, which influence aquatic food web and can pose the risk of the harmful algal blooms in the aquatic ecosystems.

Biomonitoring of aquatic ecosystems based on phytoplanktons is a routine method in European countries, on the other hand, using the biotic index is the important part of the water quality assessment. The using of biotic indices are shows the differences according to the country, climate and topography. In Turkey, using the biometric approaches for determining water quality based on phytoplankton (Çelekli and Öztürk, 2014; Toudjani et al., 2017; Çelekli and Lekesiz, 2020) was not a common method but nowadays these kind of studies become prominent.

Lake Bafa has been faced with pollution pressure due to human activities. The inflow of the Büyük Menderes River into Lake Bafa causes pollutant accumulation in the lake. Büyük Menderes River is born Dinar district of Afyon province and flows $584 \mathrm{~km}$ to the Aegean Sea. One arm of Büyük Menderes River, that seperates from the river just before it goes into the Aegean Sea, and feeds the lake. Most of the industrial, agricultural and domestic wastes of the cities around lake, accumulates in the lake. In addition Büyük Menderes River, there are also many of olive oil facilities around the lake. Accidental leakage of black water from these factories reaches the lake and also overflowing of black water wells may happen after heavy rains. On the other hand, agricultural activities around the lake, using big amount of fertilizers, pesticides and chemicals are another pollution factors on the lake. All of this organic and inorganic pollutants increase salinity levels and caused the deterioration the biodiversity. Sarı et al. (1999) reported the fish fauna of the Lake Bafa changed mainly because of the increasing salinity levels. Balık and Ustaoglu (1989) also reported that one of the third endemic species in the basin, Acanthobrama mirabilis is extinct in Lake Bafa. Previous studies (Mermer, 1989; Balık et al., 1992; Balık, 1995) reported that Cyprinus carpio, Cyprinus nasus, Barilius pectoralis, Silurus glanis were also extinct in the lake due to increased salinity levels and decreased water quality.

With this study, it is aimed to reveal the phytoplankton composition and environmental condition of Lake Bafa. The trophic and ecological status of Lake Bafa was evaluated by using of $Q$ index and TSI index based on phytoplankton and environmental data obtained monthly for two years.

\section{MATERIAL AND METHODS}

\section{Study area}

Lake Bafa, the largest lake in the Aegean Region, is located at $2 \mathrm{~m}$ a.s.l. in the inner parts of the Menteşe Mountains in the southeastern part of the Büyük Menderes basin. It is a brackish water lake with a deepest point of 21 mand a surface area of $65 \mathrm{~km} 2$. This lakeis part of the Aegean Sea in its initial formation, remains inside the coast for miles due to the alluviums carried by the Büyük Menderes River. Lake Bafa is an example of alluvial-set lakes in terms of formation.

\section{Sampling method}

The sampling was monthly carried out for 2 years in 8 sampling stations of Lake Bafa (Figure 1).

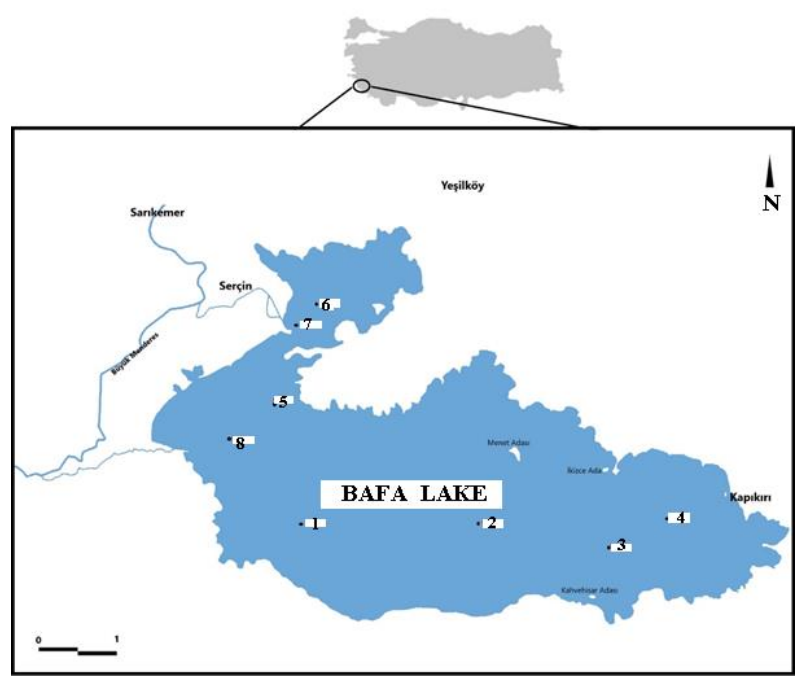

Figure 1. Sampling stations of Lake Bafa

Phytoplanton samples were collected monthly between July 2015 and June 2017 using plankton net (50 $\mu \mathrm{m}$ mesh size). Secchi disk visibility and chlorophyll a were measured in situ. Total phosphorus (TP) amount of stations were measured spectrophotometrically by using Merck Phosphate Cell Test Kit. Chlorophyll a was measured by using the BBE Moldaenke AlgaeTorch. In Lake Bafa, the water samples for quantitative analyses were fixed with lugol solution.

The quantification of microalgal cells, colonies and filamets were evaluated under inverted microscope according to the Uthermohl method (Utermohl, 1958). Counting and identification of algae were applied using a Palmer-Maloney counting cell (volume: $0.1 \mathrm{~mL}$ ) and a inverted microscope equipped with water immersion lenses and a phase-contrast attachment (400x magnification). The final abundance of each algal species was considered to be the average from two replicates. Algal species were identified according to the most 
updated literature. Taxonomy of algae was checked according to (Guiry and Guiry, 2020). Diatom identifications were performed after having prepared permanent slides following European standard NF EN 13946 (Feret et al., 2017). Diatom frustules were counted per slide at $\times 1,000$ magnification, and diatoms were identified to the lowest taxonomic level possible using taxonomic literature from central Europe (Krammer and Lange-Bertalot, 1986; Sala et al., 1993; Round and Bukhtiyarova, 1996; Krammer, 1997; Compère, 2001; Bukhtiyarova, 2006; Potapova, 2006).

\section{Statistical analysis}

The diversity scores were obtained as by using PAST3 software program.

In order to determine the trophic and ecological status of Lake Bafa, we utilized to the phytoplankton assemblage index (Q) (Padisak et al., 2006) and the Carlson Trophic State Index (TSI) (Carlson, 1977). According to Reynolds et al. (2002), on the centre of the phytoplankton functional group concept, Padisak et al., (2006) improved the $Q$ index. In order to determine ecological classes, $Q$ index grouped the lakes into five classes (Padisak et al., 2006). Q index based on the factor $F$ weights assigned to each functional group, lake type 2 (alkaline, average depth 1-3 m, and persistent) in Padisak et al., (2006) was selected because the characteristics of this type are similar to the Lake Bafa. According to Pasztaleniec and Poniewozik (2010), the $Q$ index emphasized that relative shares $(\mathrm{pi}$, where $\mathrm{pi}=\mathrm{ni} / \mathrm{N}$ ) of functional groups (ni number of the i-th functional group; $\mathrm{N}$ : total number) in the total phytoplankton numbers, and a factor number $(F)$ assessed for each functional group in each type of water body at the same time (Padisak et al., 2006).

$$
Q=\sum_{i=1}^{n} p i . F
$$

The ranges of the $Q$ index is between the $0-5$, and can be expressed into five degree classification procedure: 0-1 grade indicate bad ecological status, 1-2 grade indicate tolerable (poor) ecological status, 2-3 grade indicate medium (moderate) ecological status, 3-4 grade indicate good ecological status, 45 grade indicate high ecological status.

The TSI developed by Carlson (1977) is used to determine the productivity of lentic habitats in many limnological studies conducted today (Katip et al., 2015; Cigagna et al., 2016). According to Carlson (1977), Walker (1979), Swanson (1998) and Xu (2008), TSI proposed in order to determine the biochemical pattern of eutrophication and to powerful remove pollution related with the oligotrophic, mesotrophic and eutrophic trophic states. The ranges of the TSI is between the $0-100$, and can be expressed into four degree classification procedure. If TSI values are $<30$ or $30-40$, oligotroph, if $40-50$ is mesotroph, $50-70$ is eutroph, and $70-80$ or $>80$ is hyperutroph (Carlson and Simpson, 1996). (Table 1).
Table 1. Eutrophication state of Carlson Trophic State lindex

\begin{tabular}{lllll}
\hline Trophic state & $\mathrm{SD}(\mathrm{m})$ & $\mathrm{Chl}(\mu \mathrm{g} / \mathrm{L})$ & $\mathrm{TP}(\mu \mathrm{g} / \mathrm{L})$ & $\mathrm{TSI}$ \\
\hline Oligotrophic & $>40$ & $<2.6$ & $<12$ & $<40$ \\
Mesotrophic & $20-40$ & $2.6-7.2$ & $12-24$ & $40-50$ \\
Eutrophic & $0.5-20$ & $7.22-55.5$ & $24-96$ & $50-70$ \\
Hypertrophic & $<0.5$ & $>55.5$ & $>96$ & $>70$ \\
\hline
\end{tabular}

Secchi disk (SD), chlorophyll a (Chl a), and total phosphorus (TP) are used in the formulas for calculating the TSI (Saghi et al., 2014). The TSI index expressed by;

$$
\begin{gathered}
T S I=60-14.43 \ln (S D) \\
T S I=3056+9.81 \ln (\text { Chl } a) \\
T S I=4.14+14.43 \ln (T P)
\end{gathered}
$$

The floral simiarity between the stations were evaluated by using Bray- Curtis similarity index (Somerfield, 2008; Yoshioka, 2008). UPGMA method was used to indicate existent clustering relationships based on phytoplanktons by using PAST3 software program.

Analysis of variance (One-Way ANOVA) was applied to data for determining the statistical differences in phytoplankton species and classis, physical parameters, $Q$ index, TSI index and all diversity indices among the sampling stations of the Lake Bafa using SPSS 20.0. Pearson correlations between the physical parameters and the $Q$ index, TSI index and all diversity indices were also determined using SPSS 20.0. Canonical Correspondance Analysis (CCA) was carried out using PAST3 software programme (Ter Braak and Šmilauer, 2002) to determine the relationships between the functional groups and physical variables. CCA was carried out on the lognormal transformed abundance data. Statistical significance of the all predictor variables was assessed by Kaiser-Meyer-Olkin (KMO) Sample Proficiency Test.

\section{RESULTS}

The physical variables and coordinate of the stations indicated in Table 2. The $\mathrm{Chl}$ a values varied between 6.00 $82.8 \mu \mathrm{g} / \mathrm{L}$, the TP values varied between $1.04-1.92 \mathrm{mg} / \mathrm{L}$ and the SD values varied between $0.50-3.60 \mathrm{~m}$ at the stations in the lake. SD depth is much lower in the 5 th and 7 th stations compared to other stations. Especially in the 5th stations, the turbidity is visibly more than the other stations. Although $\mathrm{Chl}$ a values are higher in 6th and 7th stations, there is no significant difference between $\mathrm{Chl}$ a values in other stations. There is no significant difference in TP values at all stations.

As a result, a total of 63 taxa were determined from the eight sampling stations in the lake. In this study, 7 taxa belong to Cyanophyceae, 31 taxa belong to Bacillariophyceae, 15 taxa belong to Chlorophyceae, 1 taxon belong to Xanthophyceae, 7 taxa belong to Euglenophyceae and 2 taxa belong to Dinophyceae. 
Sukatar et al., Ege Journal of Fisheries and Aquatic Sciences, 38(2), 135-146 (2021)

Table 2. Mean and standart deviation values of the SD, TP, Chl-a and latitude and longitude at the stations

\begin{tabular}{lllllllll}
\hline & Station 1 & Station 2 & Station $\mathbf{3}$ & Station 4 & Station $\mathbf{5}$ & Station $\mathbf{6}$ & Station 7 & \multicolumn{1}{c}{ Station 8} \\
\hline Latitude & $37^{\circ} 29^{\prime} \mathrm{N}$ & $37^{\circ} 29^{\prime} \mathrm{N}$ & $37^{\circ} 29^{\prime} \mathrm{N}$ & $37^{\circ} 29^{\prime} \mathrm{N}$ & $37^{\circ} 31^{\prime} \mathrm{N}$ & $37^{\circ} 32^{\prime} \mathrm{N}$ & $37^{\circ} 32^{\prime} \mathrm{N}$ & $37^{\circ} 30^{\prime} \mathrm{N}$ \\
Longitude & $27^{\circ} 24^{\prime} \mathrm{E}$ & $27^{\circ} 27^{\prime} \mathrm{E}$ & $27^{\circ} 29^{\prime} \mathrm{E}$ & $27^{\circ} 31^{\prime} \mathrm{E}$ & $27^{\circ} 24^{\prime} \mathrm{E}$ & $27^{\circ} 23^{\prime} \mathrm{E}$ & $27^{\circ} 24^{\prime} \mathrm{E}$ & $27^{\circ} 22^{\prime} \mathrm{E}$ \\
$\mathrm{SD}(\mathrm{m})$ & $3.60 \pm 0.30$ & $3.40 \pm 0.50$ & $3.30 \pm 0.20$ & $3.00 \pm 0.15$ & $0.50 \pm 0.01$ & $2.00 \pm 0.15$ & $1.50 \pm 0.01$ & $2.50 \pm 0.19$ \\
$\mathrm{TP}(\mathrm{mg} / \mathrm{L})$ & $1.46 \pm 0.44$ & $1.92 \pm 0.67$ & $1.14 \pm 0.32$ & $1.74 \pm 0.52$ & $1.62 \pm 0.26$ & $1.04 \pm 0.11$ & $1.32 \pm 0.16$ & $1.13 \pm 0.09$ \\
$\mathrm{Chl} \mathrm{a}(\boldsymbol{\mu g} / \mathrm{L})$ & $12.4 \pm 2.33$ & $15.4 \pm 2.47$ & $14.5 \pm 1.95$ & $19.1 \pm 3.66$ & $6.50 \pm 0.43$ & $82.8 \pm 9.56$ & $69.0 \pm 7.98$ & $6.00 \pm 0.38$ \\
\hline
\end{tabular}

Bacillariophyceae was the dominant group in the phytoplankton composition. As a result of diagnosed phytoplankton between the stations, the maximum amount of cells were counted at 7 th station $(871.778 \mathrm{cell} / \mathrm{mL})$ while the minimum amount of the cells were counted at 8th station (20.833 cell/mL).

Percentage distribution of phytoplankton groups according to seasons is shown in Figure 2. As a result of the phytoplankton counting the dominant group is Bacillariophyceae (35\%) in summer in Lake Bafa. The second dominant group is Dinophyceae (31\%) while the third dominant group is Euglenophyceae (20\%) in the summer. As a result of the phytoplankton counting, the dominant group is also Bacillariophyceae (95\%) in the autumn, Dinophyceae (87\%) in the winter, and Xanthophyceae (81\%) in the winter in Lake Bafa. In Lake Bafa, distributions and relative occurence (\%) of the diagnosed phytoplanktons were given at Table 3 .
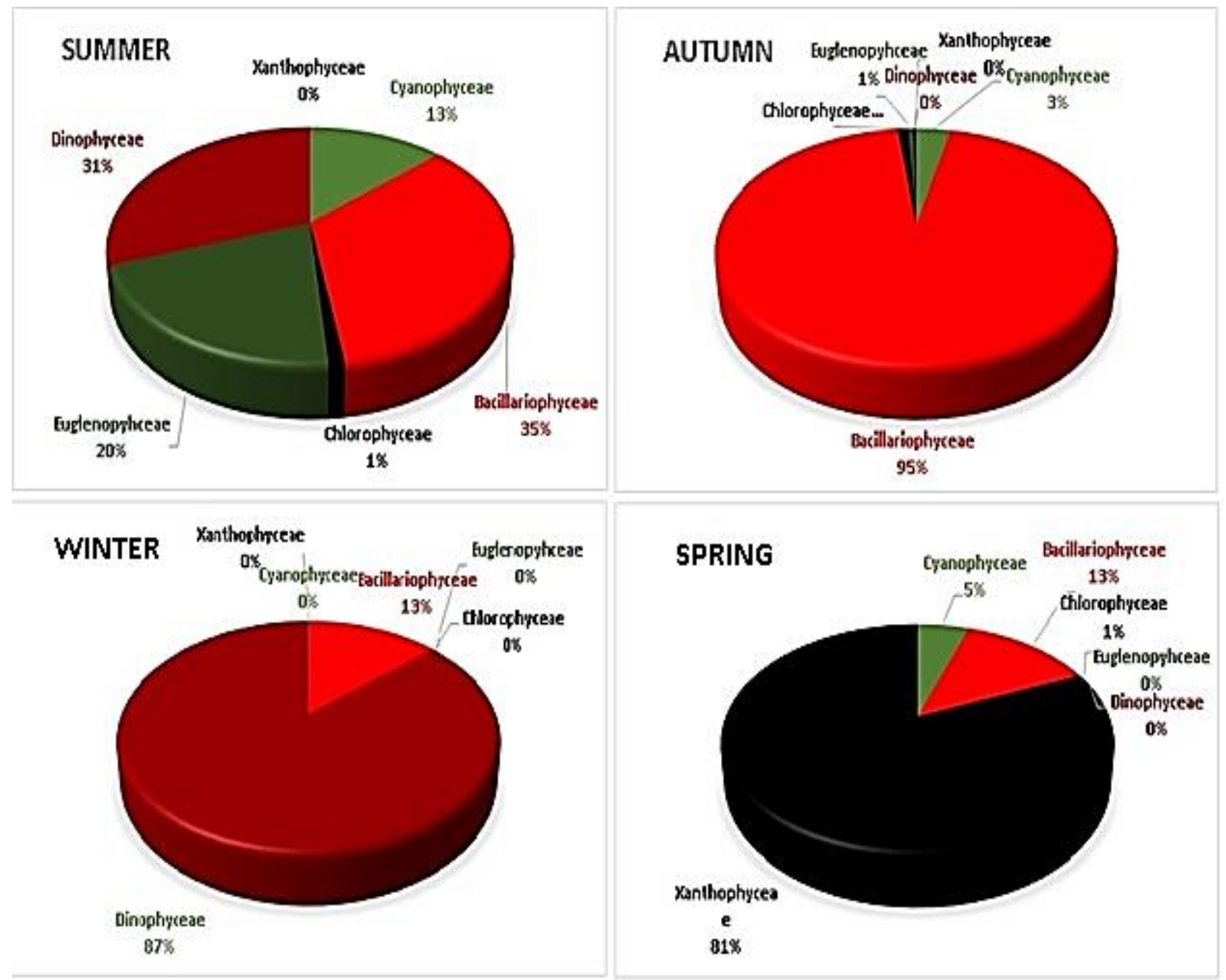

Figure 2. Proportional (\%) distributions of phytoplankton groups in abundance basis according to seasons 
Table 3. Distributions and relative occurence (\%) of phytoplankton species at the stations

\begin{tabular}{|c|c|c|c|c|c|c|c|c|c|}
\hline Code & & $\begin{array}{c}\text { Station } \\
1\end{array}$ & $\begin{array}{c}\text { Station } \\
2\end{array}$ & $\begin{array}{c}\text { Station } \\
3\end{array}$ & $\begin{array}{c}\text { Station } \\
4\end{array}$ & $\begin{array}{c}\text { Station } \\
5\end{array}$ & $\begin{array}{c}\text { Station } \\
6\end{array}$ & $\begin{array}{c}\text { Station } \\
7\end{array}$ & $\begin{array}{c}\text { Station } \\
8\end{array}$ \\
\hline & Cyanophyceae & & & & & & & & \\
\hline 1 & Komvophoron sp. & - & - & - & - & - & 0.01 & - & - \\
\hline 2 & $\begin{array}{l}\text { Cylindrospermopsis raciborskii (Woloszynska) } \\
\text { Seenayya \& Subba Raju }\end{array}$ & - & - & - & - & - & 0.68 & - & - \\
\hline 3 & Lyngbya sp. & - & - & 0.46 & - & - & - & - & - \\
\hline 4 & $\begin{array}{l}\text { Nodularia spumigena Mertens ex Bornet and } \\
\text { Flahault }\end{array}$ & 0.03 & - & 0.03 & 3.87 & 0.52 & 1.97 & 5.94 & - \\
\hline 5 & Oscillatoria spp. & 0.07 & 0.19 & 0.37 & - & 2.92 & 0.12 & 0.14 & 2.18 \\
\hline 6 & Oscillatoria limosa C.Agardh ex Gomont & - & - & - & - & 0.14 & 0.13 & 0.08 & - \\
\hline 7 & $\begin{array}{l}\text { Phormidium spp. } \\
\text { Bacillariophyceae }\end{array}$ & - & - & - & 0.07 & 1.78 & 0.10 & - & 2.08 \\
\hline 8 & Asterionella sp. & - & - & - & - & - & 0.39 & 0.10 & - \\
\hline 9 & Biddulphia sp. & - & - & 0.07 & 0.74 & - & 0.01 & 0.03 & - \\
\hline 10 & Caloneis amphisbaena (Bory) Cleve & - & - & - & 0.11 & - & - & 0.01 & - \\
\hline 11 & Campylodiscus hibernicus Ehrenberg & 0.03 & 0.01 & - & 0.03 & 0.36 & - & 0.05 & 3.24 \\
\hline 12 & Chaetoceros muelleri Lemmermann, & 17.9 & 13.7 & 5.32 & 2.61 & 0.76 & - & 0.48 & 9.06 \\
\hline 13 & $\begin{array}{l}\text { Cocconeis placentula var. euglypta } \\
\text { (Ehrenberg) Grunow }\end{array}$ & - & - & - & - & 1.69 & - & 0.12 & - \\
\hline 14 & Coscinodiscus granii L.F.Gough & 36.2 & 40.6 & 27.8 & 18.0 & 1.95 & 0.08 & 0.01 & 41.8 \\
\hline 15 & Craticula cuspidata (Kutzing) D.G.Mann & - & - & - & - & - & - & 0.01 & - \\
\hline 16 & Cyclotella sp. & - & - & 0.16 & 0.06 & - & - & - & - \\
\hline 17 & Cyclotella meneghiniana Kützing, & - & - & - & - & - & 0.34 & - & - \\
\hline 18 & Cymatopleura solea (Brébisson) W.Smith & - & - & - & - & - & 0.01 & - & - \\
\hline 19 & $\begin{array}{l}\text { Entomoneis paludosa (W.Smith) Reimer in } \\
\text { R.M.Patrick \& Reimer }\end{array}$ & - & - & - & - & - & 0.03 & - & - \\
\hline 20 & $\begin{array}{l}\text { Fragilariforma virescens var. exigua (Grunow) } \\
\text { M.Poulin in Hamilton \& al. }\end{array}$ & - & - & - & - & - & 1.44 & - & - \\
\hline 21 & Gyrosigma acuminatum (Kützing) Rabenhorst & - & - & - & - & 0.34 & 0.05 & 0.01 & - \\
\hline 22 & Gyrosigma attenuatum (Kützing) Rabenhorst & 0.06 & - & 0.04 & 2.59 & 1.18 & 0.14 & 0.16 & 0.47 \\
\hline 23 & Licmophora sp. & 0.07 & - & 0.27 & 4.20 & 12.1 & - & - & - \\
\hline 24 & Melosira nummuloides C.Agardh & 0.17 & 0.01 & 0.11 & 10.0 & 2.20 & 0.05 & 0.37 & - \\
\hline 25 & Melosira varians C.Agardh & 0.08 & - & 0.10 & 0.05 & 0.27 & 0.07 & 0.66 & 0.39 \\
\hline 26 & Navicula sp. & - & - & - & 0.04 & - & - & - & - \\
\hline 27 & Nitzschia spp. & 12.1 & 16.5 & 32.7 & 8.09 & 9.13 & 2.79 & 1.27 & 0.17 \\
\hline 28 & Nitzschia acicularis var. typica A.Cleve & - & - & - & - & 4.92 & 0.72 & 2.09 & - \\
\hline 29 & Nitzschia closterium (Ehrenberg) W.Smith & - & - & - & - & - & 0.20 & - & - \\
\hline 30 & Nitzschia incerta (Grunow) M.Peragallo & - & - & - & - & - & 0.03 & - & - \\
\hline 31 & Nitzschia intermedia Hantzsch & - & - & - & - & - & - & 0.11 & - \\
\hline 32 & Nitzschia lorenziana Grunow in Cleve \& Möller & - & 0.02 & 0.04 & - & 4.11 & 0.16 & 0.29 & - \\
\hline 33 & Nitzschia sigmoidea (Nitzsch) W.Smith & - & - & - & - & - & 0.08 & 0.12 & - \\
\hline 34 & Surirella ovalis Brébisson & - & 0.01 & - & 0.06 & 0.19 & 0.01 & 0.37 & 0.65 \\
\hline 35 & Stephanodiscus spp. & 0.29 & - & 0.29 & 0.47 & 4.34 & 2.82 & 0.49 & 1.66 \\
\hline 36 & Ulnaria ulna (Nitzsch) Compère & 0.55 & 0.31 & 0.27 & 15.7 & 36.0 & 5.04 & 3.42 & 5.65 \\
\hline 37 & Ulnaria capitata (Ehrenberg) Compère & - & - & - & - & - & - & 0.02 & - \\
\hline 38 & $\begin{array}{l}\text { Tryblionella littoralis (Grunow) D.G.Mann } \\
\text { Chlorophyceae }\end{array}$ & - & - & - & - & - & - & 0.05 & 0.09 \\
\hline 39 & Chlamydomonas sp. & - & 0.54 & - & - & - & - & - & - \\
\hline 40 & Cladophora glomerata (Linnaeus) Kützing & 0.02 & - & 0.12 & - & - & - & - & - \\
\hline 41 & Desmodesmus communis Hegewald & - & - & - & 0.01 & - & - & - & - \\
\hline 42 & Eudorina cylindrica Korshikov & - & - & - & - & - & 0.13 & - & - \\
\hline 43 & Oedogonium sp. & - & - & - & - & - & 0.03 & - & - \\
\hline 44 & Pandorina morum (O.F.Müller) Bory & - & - & - & - & - & 0.08 & 0.01 & - \\
\hline 45 & $\begin{array}{l}\text { Pseudopediastrum boryanum (Turpin) } \\
\text { E.Hegewald }\end{array}$ & - & - & - & - & - & 0.24 & 0.18 & - \\
\hline 46 & Pediastrum duplex Meyen & - & - & - & - & 0.02 & 0.06 & 0.06 & - \\
\hline
\end{tabular}


Table 3. (Continued)

\begin{tabular}{|c|c|c|c|c|c|c|c|c|c|}
\hline Code & & $\begin{array}{c}\text { Station } \\
1\end{array}$ & $\begin{array}{c}\text { Station } \\
2\end{array}$ & $\begin{array}{c}\text { Station } \\
3\end{array}$ & $\begin{array}{c}\text { Station } \\
4\end{array}$ & $\begin{array}{c}\text { Station } \\
5\end{array}$ & $\begin{array}{c}\text { Station } \\
6\end{array}$ & $\begin{array}{c}\text { Station } \\
7\end{array}$ & $\begin{array}{l}\text { Station } \\
8\end{array}$ \\
\hline 47 & Monactinus simplex (Meyen) Corda & - & - & - & - & - & 0.10 & 0.05 & - \\
\hline 48 & Scenedesmus spp. & - & - & - & - & - & 0.14 & 0.03 & - \\
\hline 49 & Tetradesmus obliquus (Turpin) M.J.Wynne & - & - & - & - & - & 0.08 & - & - \\
\hline 50 & Tetradesmus lagerheimii M.J.Wynne \& Guiry & - & 0.58 & 0.47 & - & - & - & - & - \\
\hline 51 & $\begin{array}{l}\text { Desmodesmus protuberans (F.E.Fritsch \& } \\
\text { M.F.Rich) E.Hegewald }\end{array}$ & - & - & - & - & - & 0.34 & 0.05 & - \\
\hline 52 & Spirogyra spp. & - & - & - & - & 0.13 & 0.02 & - & - \\
\hline 53 & $\begin{array}{l}\text { Stauridium tetras (Ehrenberg) E.Hegewald } \\
\text { Xanthophyceae }\end{array}$ & - & - & - & - & - & $0 . .01$ & - & - \\
\hline 54 & $\begin{array}{l}\text { Tribonema sp. } \\
\text { Euglenopyhceae }\end{array}$ & - & - & - & - & 0.09 & 0.09 & - & - \\
\hline 55 & $\begin{array}{l}\text { Lepocinclis acus (O.F.Müller) B.Marin \& } \\
\text { Melkonian }\end{array}$ & - & - & - & - & - & 3.85 & 0.09 & - \\
\hline 56 & Euglena hemichromata Skuja & - & - & - & - & - & 0.40 & 0.11 & 0.04 \\
\hline 57 & $\begin{array}{l}\text { Lepocinclis oxyuris (Schmarda) B.Marin \& } \\
\text { Melkonian }\end{array}$ & - & - & - & - & - & 2.80 & - & - \\
\hline 58 & Euglena texta (Dujardin) Hübner & - & - & - & - & - & 0.05 & 0.11 & - \\
\hline 59 & $\begin{array}{l}\text { Lepocinclis fusiformis (H.J.Carter) } \\
\text { Lemmermann }\end{array}$ & - & - & - & - & - & 3.02 & - & - \\
\hline 60 & Lepocinclis ovum (Ehrenberg) Lemmermann & - & - & - & - & - & 0.86 & - & - \\
\hline 61 & $\begin{array}{l}\text { Phacus acuminatus A.Stokes } \\
\text { Dinophyceae }\end{array}$ & - & - & - & - & - & 1.45 & - & - \\
\hline 62 & Peridinium sp. & & & & & & 0.74 & 1.26 & \\
\hline 63 & Prorocentrum cordatum (Ostenfeld) J.D.Dodge & 32.2 & 27.4 & 31.3 & 33.1 & 14.9 & 68.0 & 81.6 & 32.4 \\
\hline
\end{tabular}

As a result of the identification, it was determined that Coscinodiscus granii, Prorocentrum cordatum and Chaetoceros muelleri were dominant on station \#1 and \#8.

Coscinodiscus granii, Prorocentrum cordatum and Nitzschia spp were dominant on station \#2 and \#3. Ulnaria ulna, Prorocentrum cordatum and Coscinodiscus granii were dominant on station \#4. Ulnaria ulna, Prorocentrum cordatum and Licmophora sp. were dominant on station \#5. Ulnaria ulna, Prorocentrum cordatum and Lepocinclis acus were dominant on station \#6. Ulnaria ulna, Prorocentrum cordatum and Nodularia spumigena were dominant on station \#7 (Figure 3).

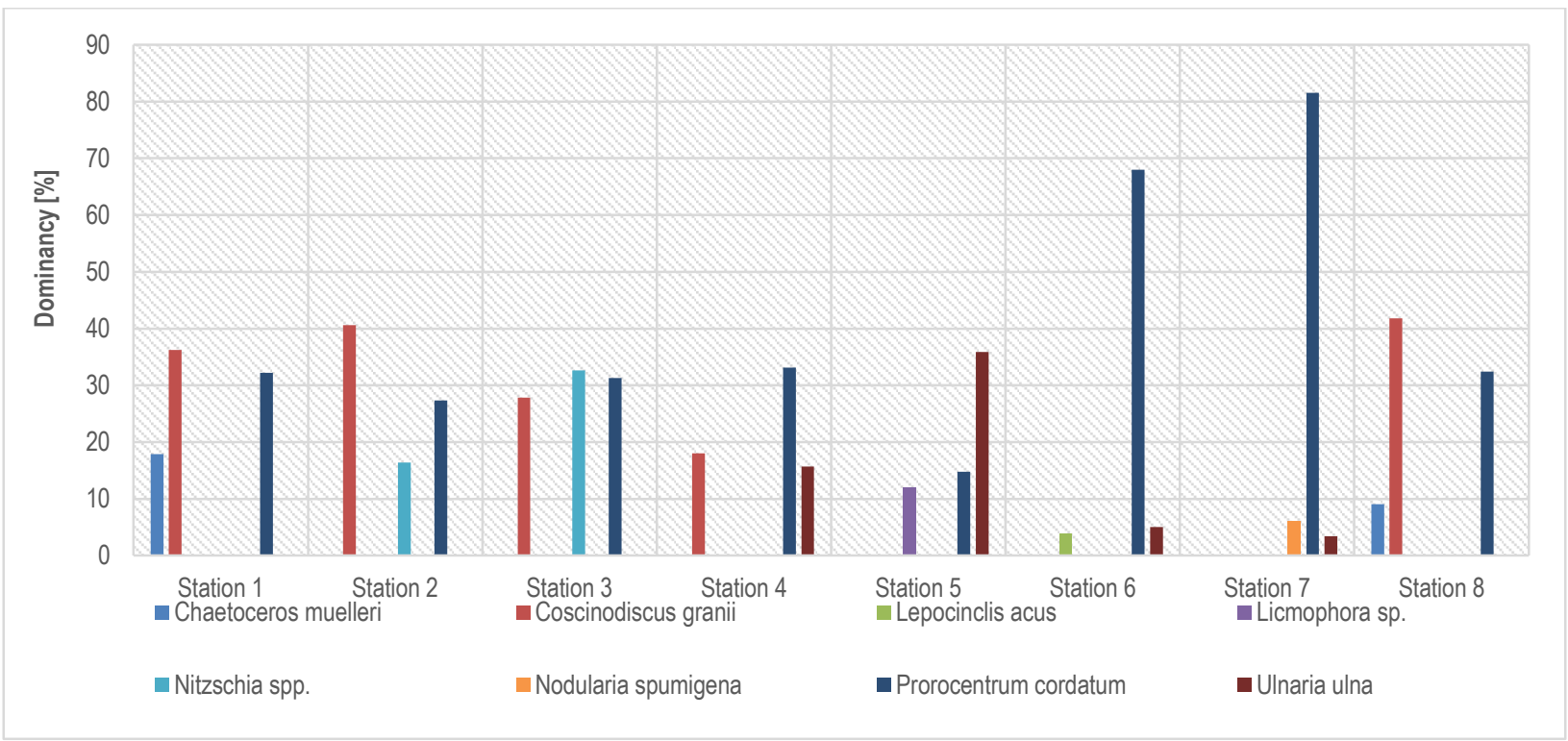

Figure 3. The most dominant phytoplankton species at the stations in Lake Bafa 
On the basis of UPGMA, the classification of the sampling stations by phytoplankton composition was defined at Figure 4. Cluster analsis was used to classify the stations detected on Lake Bafa according to phytoplankton species. As a result of the UPGMA analysis, station \#1 and station \#2 were the most similar to each other $(88 \%)$. Another high similarity was determined between the station \#2 and station \#3 (78\%).

Two biometric approaches were used for evaluating the ecological quality of Lake Bafa are illustrated in Table 4. According to the $Q$ index, the highest score value belong to station \#3. The station \#3 is determined as medium (moderate) ecological status. On the other hand, station \#6, \#7 and \#8 determined as poor ecological status. In this study, trophic characterization of Lake Bafa was determined using the Carlson Trophic State Index concerning all the TSI components: SD, Chl a amount and TP, respectively. The TSI index diversified between the scores of 49 to 59 in the spring and summer period, in Lake Bafa. This result indicated that up to the limit of mesotrophic grade (50) in terms of the Carlson model, which classifies Lake Bafa as slightly eutrophic. TSI index varied between the values 42 to 49 on winter and autumn period.

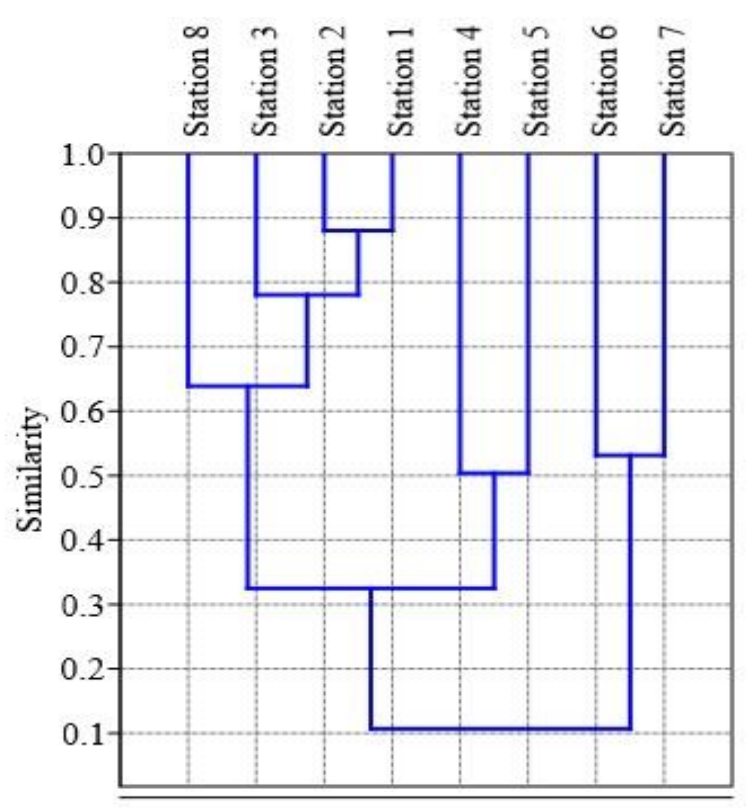

Figure 4. Classification of stations based on similarities of phytoplankton communities

Table 4. Score values of all indices and trophic status

\begin{tabular}{|c|c|c|c|c|c|c|c|c|c|}
\hline İndices & & Station 1 & Station 2 & Station 3 & Station 4 & Station 5 & Station 6 & Station 7 & Station 8 \\
\hline \multicolumn{10}{|l|}{ Biotic indices } \\
\hline Q Indice & $\begin{array}{l}\text { Score } \\
\text { Class }\end{array}$ & $\begin{array}{l}2.30 \pm 0.03 \\
\text { medium }\end{array}$ & $\begin{array}{l}2.40 \pm 0.05 \\
\text { medium }\end{array}$ & $\begin{array}{l}2.50 \pm 0.04 \\
\text { medium }\end{array}$ & $\begin{array}{l}2.20 \pm 0.06 \\
\text { medium }\end{array}$ & $\begin{array}{l}2.00 \pm 0.10 \\
\text { medium }\end{array}$ & $\begin{array}{l}1.80 \pm 0.14 \\
\text { poor }\end{array}$ & $\begin{array}{l}1.40 \pm 0.15 \\
\text { poor }\end{array}$ & $\begin{array}{l}1.60 \pm 0.15 \\
\text { poor }\end{array}$ \\
\hline TSI & $\begin{array}{l}\text { Score } \\
\text { Class }\end{array}$ & $\begin{array}{l}45 \pm 2.27 \\
\text { mesotrophic }\end{array}$ & $\begin{array}{l}43 \pm 1.65 \\
\text { mesotrophic }\end{array}$ & $\begin{array}{l}42 \pm 1.14 \\
\text { mesotrophic }\end{array}$ & $\begin{array}{l}46 \pm 1.76 \\
\text { mesotrophic }\end{array}$ & $\begin{array}{l}49 \pm 2.04 \\
\text { mesotrophic }\end{array}$ & $\begin{array}{l}51 \pm 1.56 \\
\text { eutrophic }\end{array}$ & $\begin{array}{l}59 \pm 1.88 \\
\text { eutrophic }\end{array}$ & $\begin{array}{l}53 \pm 1.03 \\
\text { eutrophic }\end{array}$ \\
\hline \multicolumn{10}{|c|}{ Species Diversity Indices } \\
\hline SDI & & $0.72 \pm 0.17$ & $0.71 \pm 0.15$ & $0.71 \pm 0.13$ & $0.81 \pm 0.16$ & $0.82 \pm 0.14$ & $0.53 \pm 0.05$ & $0.33 \pm 0.04$ & $0.71 \pm 0.12$ \\
\hline SWDI & & $1.38 \pm 0.37$ & $1.38 \pm 0.41$ & $1.41 \pm 0.33$ & $1.94 \pm 0.36$ & $2.16 \pm 0.27$ & $1.53 \pm 0.14$ & $0.91 \pm 0.019$ & $1.55 \pm 0.22$ \\
\hline MDI & & $1.26 \pm 0.33$ & $1.04 \pm 0.38$ & $1.62 \pm 0.27$ & $1.54 \pm 0.12$ & $1.91 \pm 0.33$ & $3.56 \pm 0.46$ & $2.56 \pm 0.51$ & $1.31 \pm 0.19$ \\
\hline Menhinick & & $0.08 \pm 0.01$ & $0.06 \pm 0.02$ & $0.10 \pm 0.01$ & $0.05 \pm 0.01$ & $0.07 \pm 0.02$ & $0.07 \pm 0.02$ & $0.04 \pm 0.01$ & $0.10 \pm 0.03$ \\
\hline Fisher- Alpha & & $1.40 \pm 0.21$ & $1.15 \pm 0.10$ & $1.82 \pm 0.17$ & $1.70 \pm 0.14$ & $2.14 \pm 0.25$ & $4.08 \pm 0.78$ & $2.85 \pm 0.22$ & $1.46 \pm 0.13$ \\
\hline Brillouin & & $1.38 \pm 0.33$ & $1.38 \pm 0.16$ & $1.41 \pm 0.11$ & $1.94 \pm 0.23$ & $2.16 \pm 0.14$ & $1.53 \pm 0.13$ & $0.91 \pm 0.18$ & $1.55 \pm 0.15$ \\
\hline \multicolumn{10}{|c|}{ Evenness Index } \\
\hline Evenness E1 & & $0.28 \pm 0.07$ & $0.33 \pm 0.08$ & $0.23 \pm 0.08$ & $0.37 \pm 0.09$ & $0.38 \pm 0.06$ & $0.10 \pm 0.01$ & $0.07 \pm 0.01$ & $0.34 \pm 0.06$ \\
\hline \multicolumn{10}{|c|}{ Dominancy Index } \\
\hline Berger- Parker & & $0.36 \pm 0.09$ & $0.41 \pm 0.10$ & $0.33 \pm 0.07$ & $0.33 \pm 0.07$ & $0.36 \pm 0.09$ & $0.68 \pm 0.15$ & $0.82 \pm 0.17$ & $0.42 \pm 0.11$ \\
\hline
\end{tabular}

Average \pm standard deviation

SDI: Simpson Diversity Index, SWDI: Shannon Weaver Diversity Index, MDI: Margalef Diversity Index

In this study, the SWDI, SDI, MDI, Menhinick, Brillouin and Fisher-Alpha diversity indices were calculated for each sampling station to assign species diversity. According to SWDI, the highest diversity score was found at station \#5 (2.16), while the lowest diversity scores were found at station \#1 and \#2 (1.38). According to SDI, the highest diversity score was found at station \#5 (0.82), while the lowest diversity score was found at station \#7 (0.33). According to MDI, the highest diversity score was found at station \#6 (3.56), while the lowest diversity score was found at station \#2 (1.04). According to
Menhinick Index, the highest diversity scores were found at station \#3 and \#8 (0.10), while the lowest diversity score were found at station \#7 (0.04). According to Fisher-Alpha Index, the highest diversity score was found at station \#6 (4.08), while the lowest diversity score was found at station \#2 (1.15). According to Brillouin Index, the highest diversity score was found at station \#5 (2.16), while the lowest diversity score was found at station \#7 (0.91). According to Evenness E1 index, the highest evenness score was observed at station $\# 5(0.38)$, while the lowest evenness value was seen at station \#7 (0.07). 
According to Berger-Parker index, the highest dominancy value was observed at station \#7 (0.82), while the lowest dominancy value were observed at station \#3 and \#4 (0.33) (Table 4).

Table 5, summarizes the correlations of $\mathrm{Chl}$ a, SD, TP, Q index, TSI index and species diversity indices. In this study, the random sample cases (10\% select case) were made on the biotic and diversity indices to verify datasets and to determine that the data was entered without errors in the SPSS version 20.0. The correlation between Chl- $a$ and SD ( $r=-0.305$; $p<0.01)$ was slightly strong. Between all biotic indices the highest significant correlation was determined between $Q$ and TSI $(r=0.987, p<0.01)$. TSI showed positive correlastion with both TP and chl a while it showed significant negative correlation with SD. Among species diversity indices, evenness indice and dominancy indice, the highest significant correlation was found between MDI and Fisher-Alpha $(r=1.000, p<0.01)$, SWDI and Brillouin D ( $r=1.000, p<0.01)$, SDI and Evenness $\mathrm{E} 1(r=0.926, p<0.01)$. However, an increase in the results in $Q$ index and TSI shows bad ecological quality.

Table 5. Correlation assesment between biotic and diversity indices used in Lake Bafa

\begin{tabular}{|c|c|c|c|c|c|c|c|c|c|c|c|c|c|}
\hline & SD & TP & Chl-a & $\begin{array}{c}Q \\
\text { index }\end{array}$ & TSI & SDI & SWDI & MDI & Menhinick & Fisher_alpha & Brillouin & $\begin{array}{c}\text { Evenness } \\
\text { E1 }\end{array}$ & $\begin{array}{l}\text { Berger- } \\
\text { Parker }\end{array}$ \\
\hline SD & 1 & 0.133 & $\overline{-} \cdot \overline{305}$ & 0.634 & $0 . \overline{-}$ & 0.247 & -0.29 & -0.539 & 0.268 & -0.531 & -0.29 & 0.162 & -0.428 \\
\hline TP & & 1 & 0.431 & 0.439 & $0.399^{*}$ & 0.445 & 0.314 & -0.545 & -0.511 & -0.551 & 0.314 & 0.598 & -0.396 \\
\hline Chl-a & & & 1 & -0.543 & $0.577^{\star}$ & $-.845^{\star \star}$ & -0.467 & $.906^{\star *}$ & -0.477 & $.902^{* *}$ & -0.467 & $-.900^{\star *}$ & $.907^{\star *}$ \\
\hline$Q$ index & & & & 1 & $.987^{\star \star}$ & 0.657 & 0.267 & -0.532 & 0.233 & -0.522 & 0.267 & 0.465 & $-.759^{\star}$ \\
\hline TSI & & & & & 1 & $-.720^{\star}$ & -0.33 & 0.543 & -0.328 & 0.531 & -0.33 & -0.524 & $.804^{*}$ \\
\hline SDI & & & & & & 1 & $.800^{\star}$ & -0.641 & 0.411 & -0.631 & $.800^{*}$ & $.926^{\star \star}$ & $-.959^{\star \star}$ \\
\hline SWDI & & & & & & & 1 & -0.136 & 0.145 & -0.131 & $1.000^{* *}$ & $.722^{*}$ & -0.62 \\
\hline MDI & & & & & & & & 1 & -0.287 & $1.000^{\star *}$ & -0.136 & $-.771^{*}$ & $.762^{*}$ \\
\hline Menhinick & & & & & & & & & 1 & -0.271 & 0.145 & 0.266 & -0.493 \\
\hline Fisher_alpha & & & & & & & & & & 1 & -0.131 & $-.767^{\star}$ & $.753^{*}$ \\
\hline Brillouin & & & & & & & & & & & 1 & $.722^{\star}$ & -0.62 \\
\hline Evenness E1 & & & & & & & & & & & & 1 & $-.874^{\star *}$ \\
\hline $\begin{array}{l}\text { Berger- } \\
\text { Parker }\end{array}$ & & & & & & & & & & & & & 1 \\
\hline
\end{tabular}

Phytoplankton species and three environmental variables (SD, TP and Chl a) were only used in CCA analysis according to the Kaiser-Meyer-Olkin (KMO) Sample Proficiency Test (Figure 5). The obtained results of the KMO Sample Proficiency Test were calculated as 0.744 and show that the sample size is quite good and sufficient. The CCA analysis led to the explanation total of $72 \%$ variance according to phytoplankton species.
The distributions of Lyngbya sp., Chaetoceros muelleri, Coscinodiscus granii, Nitzschia incerta and Cladophora glomerata are positively correlated to the SD. The distributions of Phormidium spp., Campylodiscus hibernicus, and Licmophora $s p$. are positively correlated to the TP. The distributions of Cylindrospermopsis raciborskii, Biddulphia sp., Navicula sp., Desmodesmus communis and Lepocinclis acus are positively correlated to the chl $a$.

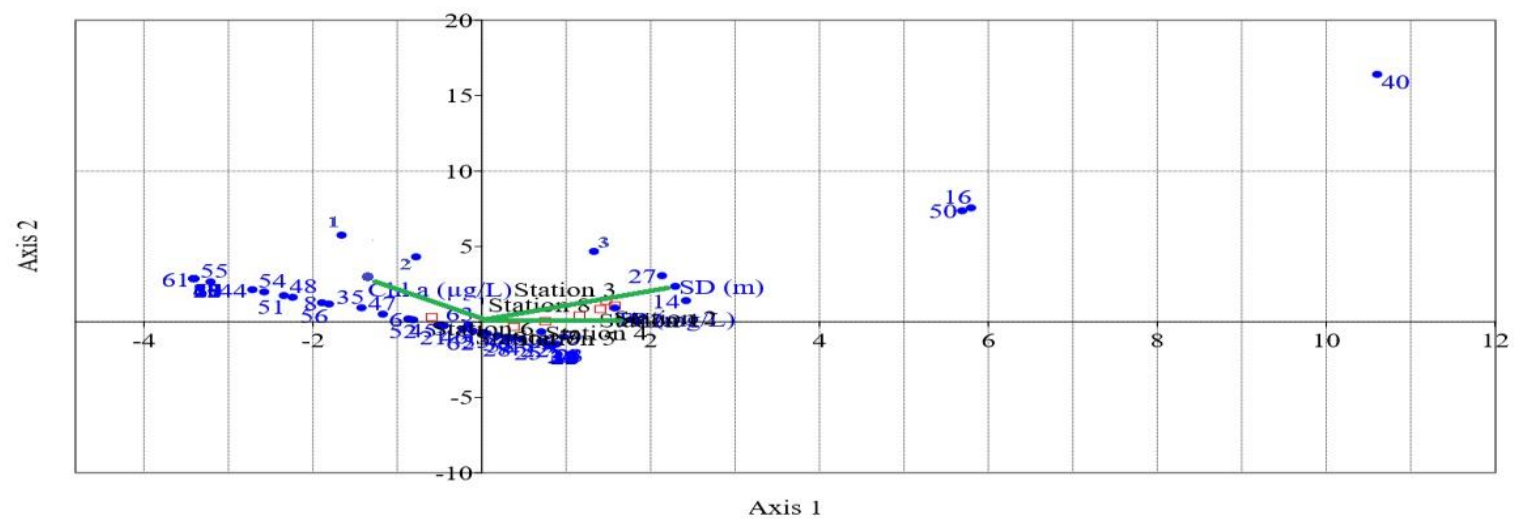

Figure 5. CCA plot of reference-, test-, and the phytoplankton species distributions with environmental variables 


\section{DISCUSSION}

In this study, total of 63 taxa were revealed during the survey. After the classification of collected samples, Cyanophyceae (11.2\%), Bacillariophyceae $(49.2 \%)$, Chlorophyceae (23.8\%), Xanthophyceae (1.5\%), Euglenophyceae $(11.2 \%)$ and Dinophyceae $(3.1 \%)$ classis were determined. Cyanobacteria members were commonly detected in eutrophic lakes in our country (Cirik-Altındağ, 1982; Sömek and Balık, 2009) and they had excessive growth in eutrophic lakes rich in nutrients in summer months (Vaitomaa, 2006). In this study, a few taxa (Cylindrospermopsis raciborskii, Lyngbya sp. and Nodularia spumigena) were also frequently encountered in inland water ecosystems in Turkey (Taş and Gönülol, 2007; Çelekli et al., 2007). Peridinium sp., was detected at the $6^{\text {th }}$ and $7^{\text {th }}$ stations of Lake Bafa, which was also found in inland water ecosystems with mesotrophic or eutrophic character in our country (Sömek et al., 2005; OngunSevindik, 2010).

The biological assessment based on phytoplankton metrics provides a more integrative approach to determine the ecological status for the conservation and restoration of the lentic ecosystems (Padisák et al. 2006; Ptacnik et al. 2009). Usages of phytoplankton indices in the evaluation of water quality have played a crucial role in water resource management worldwide (Padisák et al. 2006; Marchetto et al. 2009). Çelekli and Lekesiz (2020) reported the low cyanobacterial biovolume was found in Yapraklı Reservoir, Lake Gölhisar, and Çavdır Reservoir with good ecological conditions based on the results of the modified PTI and MedPTI. Bacillariophyta showed the highest contribution (54.6\%) to total phytoplankton community in Çavdır Reservoir. Low cyanobacteria and high diatom biovolume levels support good ecological conditions. Kazancı et al. (2008) were determined 15 species of phytoplankton from 4 different algal groups (Dinophyta, Chlorophyta, Bacillariophyta and Cyanophyta) in Lake Bafa on July 1996-1997. Although the number of Bacillariophyta species was higher than the other taxa, Dinophyta was the dominant group in the lake. According to Kazancl et al. (2008), all dominant species belong to Dinophyta. Prorocentrum minimum was the most dominant species with $78.8 \%$ (11.000 org mll$^{-1}$, organism per mililitre) of total phytoplankton whilst second dominant species was Prorocentrum micans (19.37\% of total phytoplankton and 2704.43 org ml-1). Peridinium spp was third dominant species with $1.78 \%$ (248.29 org ml-1). Kazancl et al. (2008) also calculated the diversity of the phytoplankton according to the SWDI. The diversity was found low (0.58) in the Lake Bafa because of the high quantity of Prorocentrum minimum (11 000 org $\mathrm{ml}^{-1}$ and $78.8 \%$ of total phytoplankton). In this study, all trophic components apparently were coherent in the determination of the trophic status of the Lake Bafa. Studies conducted in the lake, indicate the differences between the $\mathrm{Chl}$ a values and total phytoplankton which showed in some samples was likely due to high abundance of a broad algae masses in Lake Bafa on all seasons. Shekhar et al., (2008) reported that they determined the classis of Chrysophyceae $(2 \%)$, Cyanophyceae $(25 \%)$, Bacillariophyceae $(20 \%)$, Chlorophyceae $(36 \%)$ and Euglenophyceae $(17 \%)$ in the Bhadta Reservoir. Shekhar et al., (2008) determined in polluted zones, percentage of Cyanophyceae and Euglenophyceae was higher when compared to Chlorophyceae and Bacillariophyceae. Ochocka and Pasztalenice (2016) reported that they determined the classis of Cyanophyceae, Bacillariophyceae, Chlorophyceae, Cryptophyceae and Dinophyceae in the Masurian Lake. Ochocka and Pasztalenice (2016) stated that dominant group was filamentous cyanobacteria species (Planktothrix, Limnothrix, Planktolyngbya, Pseudoanabaena) in the lake.

Results of SD in the present study are relatively similar compared to mesotrophic lakes (Vollenweider and Kerekes, 1982). Similarly, the fact that $\mathrm{chl}$ a concentrations were determined to be moderate indicates that the moderately light transmission is not due to phytoplankton. In this study, the lakes were determined at the border of the mesotrophic state or slightly above the average TDI values calculated by using the chl $\mathrm{a}$ and SD measurement values.

In this study, the Chl a value varied between 6.00-82.8 $\mu \mathrm{g} / \mathrm{L}$ at the stations in Lake Bafa. Sakamoto (1966) reported the degree of chlorophyll a as $5.00-140 \mu \mathrm{g} / \mathrm{L}$ for eutrophic lakes, $1.00-15.0 \mu \mathrm{g} / \mathrm{L}$ for mesotrophic lakes and 0.30-2.50 $\mu \mathrm{g} / \mathrm{L}$ for oligotrophic lakes. The stations \#1, \#2, \#3, \#4, \#5 and \#8 are mesotrophic in Bafa Lake according to Sakamoto (1966) while the stations \#6 and \#7 are eutrophic in Bafa Lake.

In this study, it has been reported that the Ulnaria ulna which is frequently detected in Lake Bafa, is found in waters rich in nutritious minerals and high turbidity, and is a characteristic species of eutrophic lakes (Hustedt, 1930; Reynolds et al., 2002). Algae species, Euglena sp., Oscillatoria sp., Scenedesmus sp., and Nitzschia sp. was found on summer and autums in Lake Bafa, which are found in polluted waters (Nandan and Aher 2005; Çelekli and Lekesiz, 2020).

Çelekli et al. (2007) performed the multivariate approaches in three Aegean reservoirs. CCA analysis indicated that phytoplankton composition and distribution were mainly governed by environmental factors by TP, DO, TKN, BOD 5 , TOC and temperature. The first two CCA axes explained 31\% of cumulative percentage variance of species data with $97.7 \%$ between species-environment correlations during the study period. With regard to the ecological status, values of the MedPTI indicated good quality waters for Ayvacık and Bayramiç Reservoirs, while Sevişler Reservoir had a moderate water quality. Based on the PTI, Bayramiç and Sevişler Reservoirs were classified as or moderate ecological status, while Ayvacık Reservoir indicated a good water quality. In Lake Bafa, The CCA analysis led to the explanation total of $72 \%$ variance according to phytoplankton species. The distributions of Lyngbya sp., Chaetoceros muelleri, Coscinodiscus granii, Nitzschia incerta and Cladophora glomerata are positively correlated to the SD. The distributions of Phormidium spp., 
Campylodiscus hibernicus, and Licmophora sp. are positively correlated to the TP. The distributions of Cylindrospermopsis raciborskii, Biddulphia sp., Navicula sp., Desmodesmus communis and Lepocinclis acus are positively correlated to the chl a.

Chlorococcales in Lake Bafa was the dominant order in the second dominant group of Chlorophyceae. Likely, Scenedesmus and Pediastrum species had been found abundantly in oligomesotrophic reservoirs and eutrophic lakes in Turkey (İşbakan-Taş et al., 2002; Kıvrak and Gürbüz, 2005; Ongun-Sevindik, 2010; Çelekli et al., 2018).

Wetzel (1975) reported that members of the Euglenophyceae classis were mostly found in shallow waters rich in organic matter. The Lepocinclis fusiformis, Lepocinclis ovum and Phacus acuminatus species identified in the research area are widely found in mesotrophic or eutrophic inland water ecosystems in our country (Cirik-Altındağ, 1982; Kılınç, 1998; Ersanlı and Gönülol, 2003).

The UPGMA is a simplest method in order to constructing a tree from distance matrix which has been used often in ecology, systematics and taxonomy. The clustering technique that used in this method based on arithmetic averages of the measures of dissimilarity and similarity (James and McCulloch, 1990). In this study, the station \#1 and \#2 were the most similar to each other according to phytoplankton community. These two stations are located in the middle of the lake and they are far from the drainage points of Büyük Menderes River. Other high similarities were determined between the station \#2 and \#3 while the lowest similarities was determined between the station \#6 and \#8. The station \#2 and \#3 are located relatively close to each other.and the station \#3 is located close to the lake shore. The station \#6 and \#8 differ in depth and sediment structure. The lake is shallow and the bottom structure is muddy in the station \#6 while, the lake is relatively deep and macrophytes are predominant in the station \#8.

On the basis of diversity indices, species diversity values ranged from 0.04 to 4.08 . According to Mason (2002), SWDI values ranges from $>3$ it indictes clean water, 1-3 shows moderate pollution, and $1<$ shows severe pollution, respectively. Ghosh and Biswas (2005) reported that the diversity value ranges from 0 (low density) to 1 at the SDI. Lake Bafa is oligotrophic state according to TP values while mesotrophic-eutrophic state according to chl a values. Lakes with a SD depth of more than $4 \mathrm{~m}$ are oligotrophic and 2-4 m are mesotrophs. Average SD depth in Lake Bafa is $2.47 \mathrm{~m}$. When Lake Bafa is examined seasonally in terms of this parameter, it shows oligotroph-mesotroph lake characteristics. The TSI index results showed that Lake Bafa was from the limiting mesotrophic to slightly eutrophic. Sömek and Ustaoğlu (2016) stated that oligotrophic and mesotrophic indicatör species are found together in the phytoplankton composition of Saklıgöl, Karagöl, Gökçeova Pond and Kartal Lake, and considering the TSI values, the productivity status of the studied lakes is still oligotrophic but has a transition trend towards mesotrophic state. According to Akçaalan et al. (2007), in the research carried out in Sapanca Lake, which is an oligo-mesotrophic lake, diatom was reported to be predominantly found during the research period. According to Fakıoğlu and Demir (2011), the trophic level of Beyşehir lake is mesotrophic according to total phosphorus concentration, phytoplankton, chl a concentration value. The variation of the TP value of the Lake Bafa was examined and the TP value was found at the bottom depth and low on the surface. This is thought to be due to the release of phosphorus from the sediment. Lake Bafa is classified as mesotrophic according to the depth of Secchi. However, the low depth of Secchi may be caused by the increase of turbidity with the mixture from the bottom in shallow water. In addition, some eutrophic species have been found in the phytoplankton composition, Cyanophyceae have shown periodic increases, and the species and numbers of the Euglenophyceae groups which show organic contamination have increased.

Ongun-Sevindik et al. (2017) determined ecological status of two Mediterranean lakes with $Q$ index. The average $Q$ index was found as 3.05 for Lake Taşkısığı and 2.56 for Lake Akgöl, which reflect good and medium water qualities, respectively. The $Q$ quality index generally varied between 2 and 4 (medium to good), and it was higher during winter in both of the lakes. Values were slightly higher in Lake Taşkısığı than Lake Akgöl during the studied period ( $f=11.13, P<0.05$ ). In Lake Bafa, the highest $Q$ index score values obtained from station \#3, and the station \#3 also is determined as a moderate ecological status. In this study, the sampling stations \#6, \#7 and \#8 determined as a bad ecological status.

Considering the TDI values in the phytoplankton composition of the lake, it can be said that although the productivity status of the studied lake is still "mesotrophic", it has a tendency towards "eutrophic" state. According to the $Q$ values, the first five stations reflect the moderate ecological state, while the $6^{\text {th }}, 7^{\text {th }}$ and $8^{\text {th }}$ stations represent the poor ecological state.

\section{CONCLUSION}

The dataset obtained for two years study period in Lake Bafa indicated that the variability of trophic status of the lake. $Q$ index and the TSI index classified Lake Bafa as moderate (medium) and meso-eutrophic, respectively. According to the obtained biological dataset from Lake Bafa, it is clear that due to the pollution factors as intense domestic and industrial wastes cause pollution presure on Lake Bafa. Industrial establishments located around the lake should be inspected frequently and prevented from giving their wastes to the system without treatment. Intermittent monitoring of Lake Bafa is benefical to prevent the negative effect of pollutants. As a result of this study, there is an importantly need for the constitute of biotic indices-based phytoplankton species to assess with high accuracy freshwaters.

\section{ACKNOWLEDGEMENTS}

This research was supported by Scientific and Technological Research Council of Turkey (TUBITAK, Project no: 114Y249) 


\section{REFERENCES}

Akçaalan, R., Albay, M., Gürevin, C. \& Çevik, F. (2007). The influence of environmental conditions on the morphological variability of phytoplankton in an oligo-mesotrophic Turkish lake. International Journal of Limmnology, 43, 21-28. DOI:10.1051/limn/2007024

Balık, S. \& Ustaoglu, M.R. (1989). Bafa golundeki Ulubat baligi (Acanthobrama mirabilis Ladiges, 1960)'nin biyoekolojik ve ekonomik yonlerden incelenmesi. Turkish Journal of Zoology, 13(3), 141-174.

Balık, S. Ustaoglu, M.R. \& Sarı, H.M. (1992). Bafa gölü (Sóke-Aydın) kababurun (Chondrostoma nasus I., 1758) populasyonunun biyolojik özelliklerinin incelenmesi. Fırat Universitesi XI. Ulusal Biyoloji Kongresi, 24-27 Haziran 1992, p. 49-58, Elazığ.

Balık, S. (1995). Freshwater fish in Anatolia, Turkey. Biological Conservation (72), 213-223, Oxford. DOI: 10.1016/0006-3207(94)00084-4

Bukhtiyarova, L.N. (2006). Additional data on the diatom genus Karayevia and a proposal to reject the genus Kolbesia. Nova Hedwigia Beiheft, 130, 85-96.

Carlson, R.E. (1977). Atrophic state index for lakes. Limnology and Oceanography, 22(2), 361-369. DOI: 10.4319/lo.1977.22.2.0361

Carlson, R.E. \& Simpson, J. (1996). A coordinator's guide to volunteer lake monitoring methods. North American Lake Management Society. 96 pp.

Çelekli, A., Albay, M. \& Dügel, M. (2007). Phytoplankton (except Bacillariophyceae) Flora of Lake Gölköy (Bolu). Turkish Journal of Botany, 31, 49-65.

Çelekli, A. \& Öztürk, B. (2014). Determination of ecological status and ecological preferences of phytoplankton using multivariate approach in a Mediterranean reservoir. Hydrobiologia, 740, 115-135. DOI:10.1007/s10750-014-1948-8

Çelekli, A., Toudjani, A.A., Lekesiz, H.Ö. \& Çetin, T. (2018). Ecological quality assessment of running waters in the North Aegean catchment with diatom metrics and multivariate approach. Limnologica, 73, 20-27. DOI: $10.1016 /$ j.limno.2018.09.001

Çelekli, A. \& Lekesiz, Ö. (2020). Eco-assessment of West Mediterranean basin's rivers (Turkey) using diatom metrics and multivariate approaches. Environmental Science and Pollution Research, 27, 27796-27806. DOI:10.1007/s11356-020-09140-1

Cigagna, C., Bonotto, D.M., Camargo, A.F.M. \& Sturaro, J.R. (2016). Trophic state index (TSI) and physico-chemical characteristics of a shallow reservoir in southeast Brazil. Environmental Earth Sciences, 75(2), 1-11. DOI:10.1007/s12665-015-4951-0

Cirik-Altındağ, S. (1982). Phytoplankton of Manisa-Marmara Lake, I-Cyanophyta (in Turkish with French abstract). Doğa Bilim Dergisi, 6(3), 67-81.

Compère, $P$. (2001). Ulnaria (Kützing) Compère, a new genus name for Fragilaria subgen. Alterasynedra Lange-Bertalot with comments on the typification of Synedra Ehrenberg In: R. Jahn, J.P. Kociolek, A. Witkowski, $P$. Compère (Eds), Lange-Bertalot-Festschrift: Studies on Diatoms. Dedicated to Prof. Dr. Dr. h.c. Horst Lange-Bertalot on the occasion of his 65th Birthday. A.R.G. Gantner Verlag. K.G. pp. 97-102

Ersanlı, E. \& Gönülol, A. (2003). Study on the phytoplankton and seasonal variation of Lake Simenit (Terme-Samsun, Turkey). Turkish Journal of Fisheries and Aquatic Sciences, 3, 29-39.

European Commission (2000). Directive 2000/60/EC of the European Parliament and of the council of 23rd October 2000 establishing a framework for community action in the field of water policy. Official Journal of the European Communities, L327/1. European Commission, Brussels.

Fakıoğlu, Ö. \& Demir, N. (2011). The spatial and seasonal variations of phytoplankton biomass in Lake Beyşehir. Ekoloji, 20(80), 23-32. DOI:10.5053/ekoloii.2011.804

Feret, L., Bouchez, A. \& Rimet, F. (2017). Benthic diatom communities in high altitude lakes: a large scale study in the French Alps. Annales de Limnologie - International Journal of Limnology, 53, 411-423. DOI:10.1051/limn/2017025

Ghosh, D. \& Biswas, J. K. (2005). Macroinvertebrate diversity indices: A quantitative bioassessment of ecological health status of an Oxbow Lake in Eastern India. Journal of Advances in Enviromental Health Research, 3(2), 78- 90. DOI:10.22102/JAEHR.2015.40190

Guiry, M.D. \& Guiry, G.M. (2020). AlgaeBase. World-wide electronic publication, National University of Ireland, Galway. Retrieved from http://www.algaebase.org (25.12.2019).

Gürbüz, H. \& Altuner, Z. (2000). Palandöken (Tekederesi) Göleti fitoplankton toplulugu üzerinde kalitatif ve kantitatif bir arastırma. Turkish Journal of Biology, 24, 13-30.

Hustedt, F. (1930). Bacillariophyta Diatome Heft: 10 A Pascher DieSusswasser Flore Mitteleuropas. Ed. Gustav Fischer Pub., Jena, 466p, Germany.

İşbakan Taş, B., Gönülol, A.\& Taş, E. (2002). A study on the seasonal variation of the phytoplankton of Lake Cernek (Samsun-Turkey), Turkish Journal of Fisheries and Aquatic Sciences, 2, 1231-128.

James, F.C. \& McCulloch, C.F. (1990). Multivariate analysis in ecology and systematics: Panacea or pandora's box. Annual Review of Ecology, 21, 129-66. DOI: 10.1146/annurev.es.21.110190.001021

Katip, A., İleri, S., Karaer, F. \& Onur, S. (2015). Determination of the trophic state of Lake Uluabat (Bursa-Turkey). Ekoloji, 24(97), 24-35. DOI:10.5053/ekoloji.2015.07

Kazancı, N., Girgin, S. \& Dügel, M. (2008). Research on the limnology of Lake Bafa in South-Western Turkey and climate change impacts, Rewiew of Hydrobiology, 2, 207-223.

Kılınç, S. (1998). A study in the seasonal variation of phytoplankton in Hafik Lake (Sivas, Turkey). Turkish Journal of Botany, 22, 35-41.

Kıvrak, E. \& Gürbüz, H. (2005). Seasonal variations in phytoplankton composition and physical-chemical features of Demirdöven Dam Reservoir, Erzurum, Turkey. Biologia Bratislava, 60(1), 1-8.

Krammer, K. \& Lange-Bertalot, H. (1986-1991). Bacillariophyceae 1. Teil: Naviculaceae; 2. Teil: Bacillariaceae, Epithemiaceae, Surirellaceae; 3. Teil: Centrales, Fragilariaceae, Eunotiaceae; 4. Teil: Achnanthaceae. Kritische Ergänzungen zu Navicula (Lineolatae) und Gomphonema. Stuttgart: Fischer.

Krammer, K. (1997). Die cymbelloiden Diatomeen. Teil 2: Encyonema part., Encyonopsis und Cymbellopsis. Stuttgart: Cramer.

Marchetto, A., Padedda, B.M., Mariani, M.A., Luglie, A. \& Nicola, S. (2009). A numerical index for evaluating phytoplankton response to changes in nutrient levels in deep mediterranean reservoirs. Journal of Limnology, 68, 106-121. DOI:10.4081/jlimnol.2009.106

Mason, C.F. (2002). Biology of freshwater pollution. 4th ed. New York, NY, USA: Prentice Hall.

Mermer, A. (1989). Gediz Nehrindeki Kababurun Baligi (Chondrostoma nasus Linnaeus, 1758) populasyonunun biyolojik yöndenden incelenmesi. - Ege Üniversitesi Fen Bilimleri Enstitüsü Biyoloji Anabilim Dali, Yuksek Lisans Tezi, 48 pp, İzmir.

Nandan, S.N. \& Aher, N.H. (2005). Algal community used for assesment of water quality of Haranbaree Dam and Mosam River of Maharashtra. Journal of Environmental Biology, 26, 223-227.

Ochocka, A. \& Pasztalenice, A. (2016). Sensitivity of plankton indices to lake trophic conditions. Environmental Monitoring Assessment, 188, 622. DOI:10.1007/s10661-016-5634-3

Ongun-Sevindik, T. (2010). Phytoplankton composition of Çaygören Reservoir, Balikesir-Turkey. Turkish Journal of Fisheries and Aquatic Sciences, 10, 295-304. DOI:10.4194/trjfas.2010.0301

Ongun-Sevindik, T., Tunca, H., Gönlüol, A., Yıldırım-Gürsoy, N., Küçükkaya, Ş.N. \& Durgut-Kınalı, Z. (2017). Phytoplankton dynamics and structure, and ecological status estimation by the $Q$ assemblage index: a comparative analysis in two shallow Mediterranean lakes. Turkish Journal of Botany, 41, 25-36. DOI:10.3906/bot-1510-22

Padisak, J., Borics, G., Grigorszky, I. \& Soroczki-Pinter, E. (2006). Useofphytoplankton assemblages for monitoring ecological status of lakes within the Water Framework Directive: the assemblage index. Hydrobiologia, 553,1-14. DOI:10.1007/s10750-005-1393-9 
Pasztaleniec, A. \& Poniewozik, M. (2010). Phytoplankton based assessment of the ecological status of four shallow lakes (Eastern Poland) according to Water Framework Directive - a comparison of approaches. Limnologica, 40, 251-259. DOI:10.1016/j.limno.2009.07.001

Pasztaleniec, A. (2016). An advanced phytoplankton trophic index: Test and validation with a nationwide lake survey in Poland. International Review of Hydrobiology, 101, 20-35. DOl:10.1002/iroh.201501799

Potapova, M. (2006). Achnanthidium zhakovschikovii sp. nov. (Bacillariophyta) and related species from rivers of Northwestern Russia. Nova Hedwigia, 82, 399-408. DOI:10.1127/0029-5035/2006/0082-0399

Ptacnik, R, Solimini, A.G. \& Brettum P. (2009). Performance of a new phytoplankton composition metric along a eutrophication gradient in Nordic lakes. Hydrobiologia, 633, 75-82. DOI:10.1007/s10750-009-9870-1

Reynolds, C.S., Huszar, V., Kruk, C., Naselli-Flores, L. \& Melo, S. (2002). Rewiew, towards a functional classification of the freshwater phytoplankton. Journal of Plankton Research, 24(5), 417-428. DOI:10.1093/plankt/24.5.417

Round, F.E. \& Bukhtiyarova, L. (1996). Four new genera based on Achnanthes (Achnanthidium) together with a re-definition of Achnanthidium. Diatom Research, 11, 345-361. DOI: 10.1080/0269249X.1996.9705389

Saghi, H., Karimi, L. \& Javid, A. H. (2014). Investigation on trophic state index by artificial neural networks (case study: Dez Dam of Iran). Applied Water Science, 5(2), 2190-5487. DOI:10.1007/s13201-014-0161-2

Sakamoto, M. (1966). The chlorophyll amount In the euphotic zone in some Japanese Lakes and its significace In thr photosynthetic production of phytoplankton community. The botanical magazine Tokyo, 79, 77-78. DOI: $10.15281 /$ jplantres1887.79.77

Sala, S. E., Guerrero, J. M. \& Ferrario, M. E. (1993). Redefinition of Reimeria sinuata (Gregory) Kociolek \& Stoermer and recognition of Reimeria uniseriata nov. spec. Diatom Research, 8, 439-446. DOI: 10.1080/0269249X.1993.9705273

Sarı, H.M., Balık, S., Bilecenoğlu, M. \& Türe, G. (1999). Recent changes in the fish fauna of Lake Bafa, Aegean region of Turkey. Zoology in the Middle East, 18, 67-76. DOI: 10.1080/09397140.1999.10637783

Shekhar, T.R., Kiran, B.R., Puttaiah, E.T., Shivaraj, Y. \& Mahadevan, K.M. (2008). Phytoplankton as index of water quality with reference to industrial pollution. Journal of Environmental Biology, 29(2), 233-6.

Sömek, H., Balık, S. \& Ustaoğlu M.R. (2005). Phytoplankton of Topçam Dam Lake (Çine-Aydın) and their seasonal variations (in Turkish with English abstract). Süleyman Demirel University Eğirdir Journal of Fisheries Faculty, 1(1), 26-32.

Sömek, H. \& Balık, S. (2009). Seasonal variation of algal flora and environmental conditions of Karagöl (A Mountain Lake, İzmir-Turkey) (in
Turkish with English abstract). Ege Journal of Fisheries and Aquatic Sciences, 26(2), 121-128. DOI: 10.12714/egejfas.2016.33.2.05

Sömek, H. \& Ustaoğlu M.R. (2016). Summer phytoplankton composition and trophic state index values of some mountain lakes in Western Anatolia (Denizli-Muğla). Ege Journal of Fisheries and Aquatic Sciences, 33(2), 121-128. DOI:10.12714/egejfas.2016.33.2.05

Somerfield, P. J. (2008). Identification of the Bray-Curtis similarity index: Comment on Yoshioka (2008). Marine Ecology Progress Series, 372, 303-306. DOI: $10.3354 /$ meps07841

Swanson, E. R. (1998). Trophic state index revisited. Lake Line, 18(4), 18-20.

Taş, B. \& Gönülol, A. (2007). Planktonic algae of Derbent Dam Lake (Samsun, TURKEY). Journal of FisheriesSciences.com, 1(3), 111-123. DOI: $10.3153 /$ jfscom.2007014

Ter Braak, C.J.F. \& Smilauer, P. (2002). CANOCO reference manual and CanoDraw for Windows user's guide: Software for Canonical Community Ordination (version 4.5). Biometris, Wageningen.

Toudjani, A.A., Çelekli, A., Gümüş, E.Y., Kayhan, S., Lekesiz, H.Ö. \& Çetin, T. (2017). A new diatom index to assess ecological quality of running waters: a case study of water bodies in western Anatolia. Annales de Limnologie - International Journal of Limnology, 53, 333-343.

DOI:10.1051/limn/2017012

Utermohl, H. (1958) Zur ver vollkommung der quantitativen phytoplanktonmethodik. Mitteilung internationale vereinigung fuer theoretische unde amgewandte limnologie, 9, 39 p. DOI: 10.1080/05384680.1958.11904091

Vaitomaa, J. (2006). The effects of environmental factors on biomass and microcystin production by the freshwater cyanobacterial genera Microcystis and Anabaena. Edita, Helsinki, Finland, $56 \mathrm{p}$.

Vollenweider, R.A. \& Kerekes J.J. (1982). Eutrophication of waters. Monitoring, assessment and control. OECD Cooperative programme on monitoring of inland waters (Eutrophication control), Environment Directorate, OECD, Paris. $154 \mathrm{p}$

Walker, W.W. (1979). Use of hypolimnetic oxygen depletion rate as a trophic state index for lakes. Water Research, 15(6), 1463-1470. DOI: 10.1029/WR015i006p01463

Wetzel, R.G. (1975). Limnology. W,B. Sounders Conpany, Phiadelphia, 743p.

Yoshioka, P. M. (2008). Misidentification of the Bray-Curtis similarity index. Marine Ecology Progress Series, 368, 309-310. DOI:10.3354/meps07728

Xu, F.L. (2008). Trophic classification for lakes. In: Jørgensen SE, Fath BD (eds) Ecological indicators, Vol. 5 of encyclopedia of ecology, 5 th edn., $359 p p$. 